\title{
Improved Quantum Evolutionary Computation Based on Particle Swarm Optimization and Two-Crossovers *
}

\author{
DUAN Hai-Bin(段海滨)**, XING Zhi-Hui(邢智慧) \\ School of Automation Science and Electrical Engineering, Beijing University of Aeronautics and Astronautics, \\ Beijing 100191
}

(Received 25 August 2009)

\begin{abstract}
A quantum evolutionary computation (QEC) algorithm with particle swarm optimization (PSO) and twocrossovers is proposed to overcome identified limitations. PSO is adopted to update the Q-bit automatically, and two-crossovers are applied to improve the convergence quality in the basic QEC model. This hybrid strategy can effectively employ both the ability to jump out of the local minima and the capacity of searching the global optimum. The performance of proposed approach is compared with basic QEC on the standard unconstrained scalable benchmark problem that numerous hard combinatorial optimization problems can be formulated. The experimental results show that the proposed method outperforms the basic QEC quite significantly.
\end{abstract}

PACS: 03.67.Ac, 12.20.Ds, 87.19. Lv

Quantum computation has promised prodigious powers in recent years. Its basic currency, the Q-bit, exists in an $\mathrm{ON}$ or OFF verge, which is unknown until it is read out. Therefore, operating on $k$ Q-bits, a potentially vast space of $2^{k}$ values opens up for computation which allows many computing problems to be solved simultaneously, saving time. The fundamental operation on Q-bits is a rotation, with logic gates used to combine the rotations. Particle swarm optimization (PSO) is an evolutionary computation technique inspired by observing the bird flocking or fish school, which was firstly developed by Kennedy and Eberhart ${ }^{[1]}$ in 1995 . The concept of the genetic algorithm (GA) was put forward by Holland in the 1970s to study the self adaptation behavior of natural systems. ${ }^{[2]}$ GA has a limited population size and can be susceptible to the problems of premature convergence and stagnation, which limit its wide applications in various fields. In contrast, Q-bit chromosomes enjoy a rapidly growing population and strong randomization. To overcome the shortcomings of GA, a quantum evolutionary computation (QEC) algorithm is proposed based on the concept and principles of quantum computing. In QEC, Q-bit chromosomes, which can represent a linear superposition of solutions, are adopted to preserve the solution diversity and overcome premature. At the same time, a quantum rotation gate, which make full use of the information of the current best individual, is employed to update the individual and avoid stagnation. ${ }^{[3]}$

The basic QEC uses Q-bit gate rotation in mutation and whole interference in crossover. ${ }^{[4]}$ By using a rotation operation, the information of the currently best individual can be fully utilized to conduct the next searching process, and the whole interference can avoid prematurity. In this way, the global search capacity can be significantly improved, while the convergence speed is slowed down. In order to further improve the whole performance of QEC, a hybrid strategy is proposed in this work.

In QEC, a qubit chromosome as a string of $n$ qubits can be defined as follows: ${ }^{[5]}$

$$
q=\left[\begin{array}{cccc}
\alpha_{1} & \alpha_{2} & \cdots & \alpha_{m} \\
\beta_{1} & \beta_{2} & \cdots & \beta_{m}
\end{array}\right]
$$

where $\left|\alpha_{i}\right|^{2}+\left|\beta_{i}\right|^{2}=1, i=1, \ldots, m, m$ is the number of qubits and also the string length of the qubit individual; $\left|\alpha_{i}\right|^{2}$ gives the probability that the qubit will be found in the state of " 0 ", and $\left|\beta_{i}\right|^{2}$ gives the probability that the qubit will be found in the " 1 " state. A qubit chromosome is able to represent a linear superposition of all possible solutions. It has a better diversity characteristic than a classical chromosome. ${ }^{[6]}$ The process to get a classical chromosome is to select a random number between 0 and 1 . If it is larger than $\left|\alpha_{i}\right|^{2}$, this bit in classical chomonsome is 1 . Otherwise, 0 is chosen.

The standard mutation operation is totally random without any directions, and the speed of convergence is slowed down. However, in QEC, the qubit representation can be regarded as a mutation operator. Directed by the current best individual, quantum mutation is completed through the quantum rotation gate $U(\theta)$, and $\left[\alpha_{i} \beta_{i}\right]^{T}$ can updated with the following equation,

$$
\left[\begin{array}{c}
\alpha_{i}^{\prime} \\
\beta_{i}^{\prime}
\end{array}\right]=\left[\begin{array}{cc}
\cos \theta_{i} & -\sin \theta_{i} \\
\sin \theta_{i} & \cos \theta_{i}
\end{array}\right]\left[\begin{array}{c}
\alpha_{i} \\
\beta_{i}
\end{array}\right]
$$

* Supported by the National Natural Science Foundation of China under Grant Nos 60975072 and 60604009, Aeronautical Science Foundation of China under Grant Nos 2008ZC01006 and 2006ZC51039, and Beijing NOVA Program Foundation under Grant Nos 2007 A017.

**Email: hbduan@buaa.edu.cn

(C) 2009 Chinese Physical Society and IOP Publishing Ltd 
Figure 1 describes the polar plot of the rotation operation on a qubit ${ }^{[8]}$. It explains why the rotation gate can increase the speed of convergence.

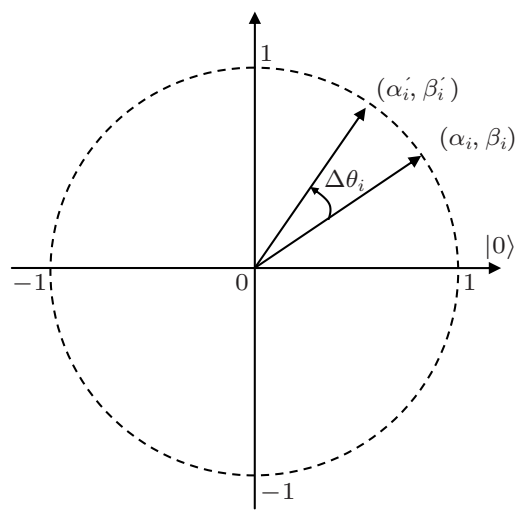

Fig. 1. Polar plot of the rotation gate for qubit chromosome.

Table 1. Rotation angle.

\begin{tabular}{ccccccc}
\hline \multirow{2}{*}{$x_{i}$ best $_{i} f(x)>f($ best $)$} & \multicolumn{5}{c}{$\theta_{i}$} \\
\cline { 3 - 7 } & $\alpha_{i} \beta_{i}>0$ & $\alpha_{i} \beta_{i}>0$ & $\alpha_{i} \beta_{i}>0$ & $\alpha_{i} \beta_{i}>0$ \\
\hline 0 & 0 & False & 0 & 0 & 0 & 0 \\
0 & 0 & True & 0 & 0 & 0 & 0 \\
0 & 1 & False & 0 & 0 & 0 & 0 \\
0 & 1 & True & $-0.05 \pi$ & $0.05 \pi$ & $\pm 0.05 \pi$ & 0 \\
1 & 0 & False & $-0.05 \pi$ & $0.05 \pi$ & $\pm 0.05 \pi$ & 0 \\
1 & 0 & True & $0.05 \pi$ & $-0.05 \pi$ & 0 & $\pm 0.05 \pi$ \\
1 & 1 & False & $0.05 \pi$ & $-0.05 \pi$ & 0 & $\pm 0.05 \pi$ \\
1 & 1 & True & $0.05 \pi$ & $-0.05 \pi$ & 0 & $\pm 0.05 \pi$ \\
\hline
\end{tabular}

Table 1 provides a convenient database for selecting the correct $\theta_{i}$, which is determined by both the quantum and classical chromosome, where $x_{i}$ is the $i$ th bit of the current classical chromosome, best ${ }_{i}$ is the $i$ th bit of the current best classical chromosome, $f(x)$ is the adaptation function. ${ }^{[7]}$

This type of crossover operation is constructed using the interference characteristic of a qubit. ${ }^{[9]}$ Table 2 shows the type of crossover operation.

Table 2. The whole interference crossover operation.

\begin{tabular}{cccccccc}
\hline 1 & $A(1)$ & $E(2)$ & $D(3)$ & $C(4)$ & $B(5)$ & $A(6)$ & $E(7)$ \\
\hline 2 & $B(1)$ & $A(2)$ & $E(3)$ & $D(4)$ & $C(5)$ & $B(6)$ & $A(7)$ \\
3 & $C(1)$ & $B(2)$ & $A(3)$ & $E(4)$ & $D(5)$ & $C(6)$ & $B(7)$ \\
4 & $D(1)$ & $C(2)$ & $B(3)$ & $A(4)$ & $E(5)$ & $D(6)$ & $C(7)$ \\
5 & $E(1)$ & $D(2)$ & $C(3)$ & $B(4)$ & $A(5)$ & $E(6)$ & $D(7)$ \\
\hline
\end{tabular}

The whole interference crossover operation can make full use of the information in the chromosome, ${ }^{[10]}$ improve the unilateralism of classical crossover and avoid premature convergence and stagnation problems.

In a quantum mutation operation, the rotation angle is a fixed number, independent of whether the individual is better or worse. ${ }^{[11]}$ When the whole interference crossover operation tries to produce a new solution to avoid premature convergence, this is undesirable for maintaining current good solutions, and the convergence speed of QEC is decreased. To improve the convergence speed and avoid premature convergence, we propose a hybrid QEC. The result is extraordinary both in theory and experiments.

In our proposed hybrid QEC, we use PSO to optimize the angle of the rotation gate in quantum mutation operation and adopt two-crossover operations. The philosophy behind the original PSO is to learn from individual's own experience (personal best solution) and the best individual experience (global best solution) in the whole swarm. Rotation angle is determined by the following expression:

$$
\theta=\frac{\theta_{\max } \cdot\left|\eta_{1} \cdot \operatorname{rand}_{1} \cdot\left(P_{i d}-X_{i d}\right)+\eta_{2} \cdot \operatorname{rand}_{2} \cdot\left(P_{g d}-X_{i d}\right)\right|}{\left(\eta_{1}+\eta_{2}\right) \cdot\left(X_{\max }-X_{\min }\right)}
$$

where $\eta_{1}$ is the cognition learning factor and $\eta_{2}$ is the social learning factor, usually $\eta_{1}=\eta_{2}=2$. In some cases, $\eta_{1}$ equals to $\eta_{2}$ and ranges from $[0,4] ; \operatorname{rand}_{1}$ and $\operatorname{rand}_{2}$ are the random numbers uniformly distributed in $[0,1]$. In this expression, we take the difference between the current solution and the best solution into consideration. This expression ensures that the rotation angle is between $\theta_{\max }$ and 0 . The angle is smaller if the current solution is better. In this operation, the current fitness and the best fitness are involved. In this way, the choice of angle is more reasonable. The process of the evolution is optimized, and the convergence speed is also improved.

The first crossover operation is the classical single point crossover. A roulette selection operation is used to choose two different quantum chromosomes from the parent generations. The child generation is then produced by crossover. After this process, two better individuals can be chosen for the next generation by evaluating their fitness. This operation is mainly to improve the convergence speed and preserve the instructive information. Typically, we choose 0.6 to 0.9 as the crossover probabilities in this process.

The fitness of the whole population can be evaluated by choosing the best one as the mutation director. In this operation, we evaluate the current quantum chromosome and use the rules shown in Table 1 to choose the direction of rotation angle. The angle value can be determined by the PSO, mentioned previously. We choose 0.01 to 0.2 as the mutation probabilities. Although it is not traditional mutation and the individual can also converge by this operation, the mutation probability cannot be very high, in each generation, only one best individual can be chosen. Meanwhile, a quantum chromosome is also used, and the selection is random. Usually, we are not sure if the "best solution" we choose is actually the global best result. Furthermore, too much prob- 
ability is not good for evolution. Another crossover operation, the whole interference crossover operation, is adopted to prevent premature convergence. It can bring new individuals to help the population jump out of premature convergence. We also choose 0.01 to 0.2 as the crossover probability. Because it is used solely to avoid premature convergence, we cannot use it in high frequency.

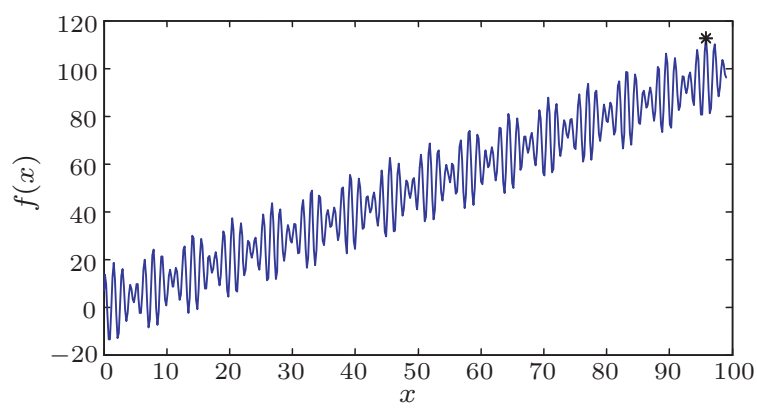

Fig. 2. Final chromosomes position of the basic QEC (marked with asterisk).

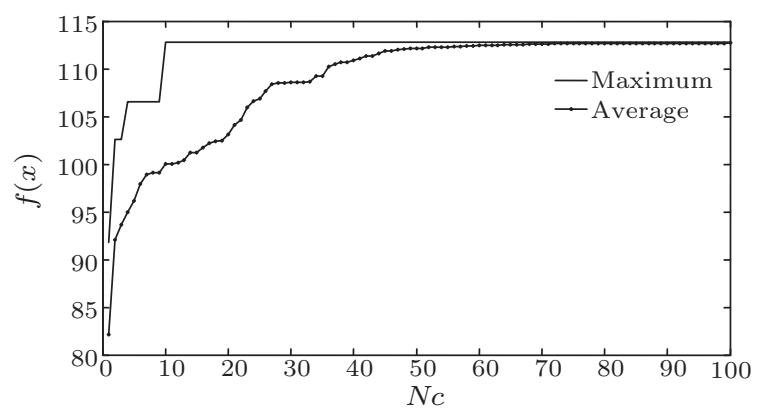

Fig. 3. The corresponding maximum and average evolution curves of the basic QEC.

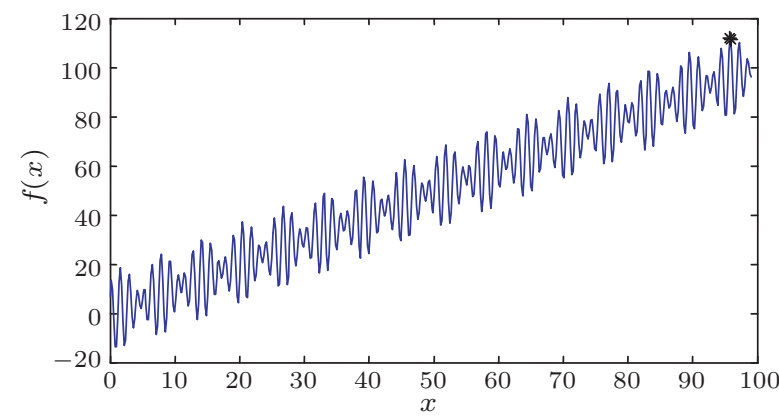

Fig. 4. Final chromosomes position of the improved QEC based on PSO and two-crossovers (marked with asterisk).

In order to investigate the feasibility and effectiveness of the proposed hybrid QEC with PSO and twocrossovers, a series of experiments are conducted on the following Benchmark problem:

$$
\operatorname{Max} f(x)=x+10 \sin (5 x)+7 \cos (4 x) .
$$

The original chromosomes are produced with strong randomization, and $n=10, N=5, L=22, P_{c c}=0.9$, $P_{i c}=0.2, P_{m}=0.2, N c_{\max }=100$. Figure 2 shows the final chromosomes position of the basic QEC with fixed rotation angle. It is apparent that it is easy to fall into premature convergence. The corresponding maximum and average evolution curves of the basic QEC are presented in Fig. 3.

Figure 4 shows the final chromosomes position of the improved QEC based on PSO and two-crossovers, and Fig. 5 shows the corresponding maximum and average evolution curves of the proposed QEC.

It is obvious that our proposed QEC model can find better solutions than the basic QECs in solving combinatorial optimization problems, and the improved QEC model can avoid premature convergence, which usually occurs in the basic QEC model.

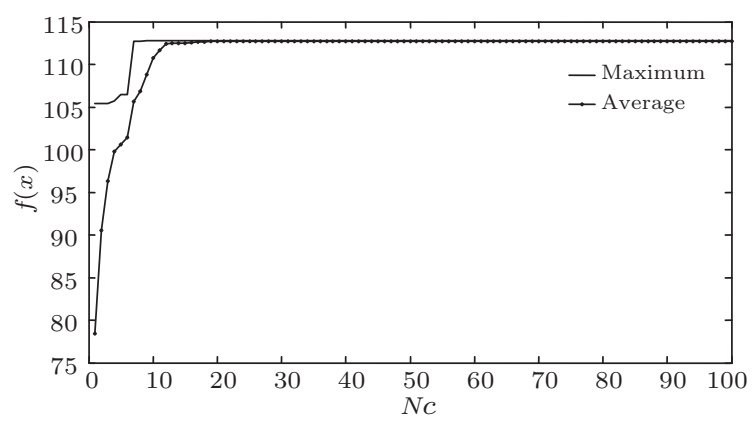

Fig. 5. The corresponding maximum and average evolution curves of the improved QEC based on PSO and two-crossovers.

In summary, our proposed QEC with PSO and two-crossovers has much better performance, with strong ability to find the optimal solution and quick convergence speed. Our future work is to investigate how to implement our method by a real quantum machine.

\section{References}

[1] Kennedy J and Eberhart R 1995 IEEE International Conference on Neural Networks (Perth, Western Australia 27 November-1 December) 41942

[2] Holland J 1975 Adaptation in Natural and Artificial Systems (Michigan: The University of Michigan Press) p 86

[3] Walther P, Resch K J, Rudolph T, Schenck E, Weinfurter $\mathrm{H}$, Vedral V, Aspelmeyer M and Zeilinger A 2005 Nature 434169

[4] Yang S Y, Liu F, and Jiao L C 2001 Acta Electron. Sin. 291873 (in Chinese)

[5] Zhang W F, Shi Z K, and Luo Z Y 2008 International Joint Conference on Neural Networks (Hongkong 1-6 June 2008) p 1510

[6] Tayarayi M H N and Akbarzadeh M R T 2007 IEEE Congress on Evolutionary Computation (Singapore 25-28 September 2007) p 2670

[7] Xiao J, Yan Y P, Lin Y, Yuan L and Zhang J 2008 IEEE Congress on Evolutionary Computation (Hongkong 1-6 June 2008) p 1513

[8] Wei M, Li Y X, Jiang D Z, He Y F, Huang X Y and $\mathrm{Xu} \mathrm{X} 2008$ IEEE Congress on Evolutionary Computation (Hongkong 1-6 June 2008) p 1722

[9] Al-Rabadi A N 2009 Int. J. Intelligent Computing and Cybernetics 252

[10] Xing Z H, Duan H B and Xu C F 2009 Lecture Notes in Computer Science $\mathbf{5 5 5 1} 735$

[11] Wang A M 2002 Chin. Phys. Lett. 19620 